\title{
Indirect transitions of a signal interacting with a moving refractive index front
}

\author{
Michel Castellanos Muñoz ${ }^{1}$, Alexander Yu. Petrov ${ }^{1}$, Liam O’Faolain ${ }^{2}$, Juntao $\mathrm{Li}^{3}$, Thomas F. \\ Krauss $^{4}$, and Manfred Eich $^{1 *}$ \\ 1 Institute of Optical and Electronic Materials, Hamburg University of Technology, Hamburg \\ 21073, Germany \\ 2 SUPA, School of Physics and Astronomy, University of St. Andrews, St. Andrews, Fife KY16 \\ 9SS, United Kingdom \\ 3 State Key Laboratory of Optoelectronic Materials \& Technology, Sun Yat-Sen University, \\ Guangzhou 510275, China \\ 4 Department of Physics, University of York, Heslington, York, YO10 5DD, United Kingdom
}

\begin{abstract}
The dynamic manipulation of light can be achieved by the interaction of a signal pulse propagating through or reflected from a refractive index front. Both the frequency and the wave vector of the signal are changed in this case, which is generally referred to as an indirect transition. We have developed a theory to describe such transitions in integrated photonic crystal waveguides. Through indirect transitions, the following effects can be envisaged: large frequency shifts and light stopping and order of magnitude pulse compression and broadening without center frequency shift. All effects can be potentially realized with a refractive index modulation as small as 0.001 .

For the experimental realization, we have used slow light photonic crystal waveguides in silicon. The refractive index front was obtained by free carriers generation with a switching pulse co-propagating with the signal in the same slow light waveguide. The group velocities of the signal and the front could be varied arbitrarily by choosing the right frequencies of the signal and switching pulses. The indirect transition was unambiguously demonstrated by considering two situations: a) the front overtaking the signal and b) the signal overtaking the front. In both cases, a blue shift of the signal frequency was observed. This blue shift can only be explained by the occurrence of the expected indirect transition and not by a direct transition without wave vector variation.
\end{abstract}

Keywords: indirect transition, dynamic frequency shift, photonic crystal waveguides, on-chip, silicon photonics

\section{INTRODUCTION}

The process of an optical signal undergoing a transition between two modes of a photonic structure is referred to as a photonic transition [1-5]. Photonic transitions can be direct, if the optical signal experiences a shift in frequency but not in wave vector, or indirect, if both frequency and wave vector of the signal are changed.

It was previously shown that light confined to a photonic structure can be shifted in frequency if a refractive index change is applied to the entire structure while the light is still confined within it. Such a process has been referred to as dynamic or adiabatic control of light in the literature [6-10]. The magnitude of the resulting optical frequency shift is proportional to the induced index change $|\Delta \mathrm{n}|$ and was first shown for light travelling in a millimeter-long semiconductor slab [11] and later for light confined in microphotonic resonators [6-8] and photonic crystal waveguides [9, 10]. These cases can be classified as direct photonic transitions, since the refractive index change causes a transition in frequency $\omega$ but leaves the wave vector $\mathrm{k}$ of the light signal unaltered. The required fast change of refractive index is achieved in these cases by generating free carriers in silicon, which leads to a refractive index change via the carrier plasma dispersion effect [12]. Different methods for generating the free carriers have been proposed and implemented, for instance by electrical injection with a p-i-n junction [13], by the linear absorption of an optical switching pulse which is incident on the device from the top [11, 7, 9, 10], and by nonlinear two-photon absorption of an optical switching pulse which co-propagates with the signal through the photonic structure, for the first time realized by us [8].

*m.eich@tuhh.de; phone +49 4042878 3147; www.tuhh.de /oem

Active Photonic Materials VI, edited by Ganapathi S. Subramania, Stavroula Foteinopoulou, Proc. of SPIE Vol. 9162, 91621W · C 2014 SPIE · CCC code: 0277-786X/14/\$18 · doi: 10.1117/12.2063078 
Indirect photonic transitions, on the other hand, imply both a change of frequency and wave vector of the optical signal, and are not only of fundamental physical interest, but may constitute, for instance, the operating principle for the realization of integrated non-reciprocal optical isolators without using magneto-optical or non-linear effects [4]. Further, they offer exciting opportunities for ultrafast delay applications $[5,14]$. In this context, indirect photonic transitions between modes that belong to different photonic bands, called indirect interband transitions, have attracted attention, since a cascade of transitions, and thus the appearance of multiple frequency components, can be avoided [15, 2-4].

Indirect photonic transitions can be achieved in different ways. For example, it was shown that an indirect photonic transition between two modes $\left(\omega_{0}, \mathrm{k}_{0}\right)$ and $\left(\omega_{1}, \mathrm{k}_{1}\right)$ separated by a frequency $\Omega=\omega_{1}-\omega_{0}$ and a wave vector $\mathrm{q}=\mathrm{k}_{1}-\mathrm{k}_{0}$ can be achieved by modulating the refractive index of the structure both temporally with a frequency $\Omega$, and spatially with a spatial frequency q $[1-4,15]$. This process can be explained as a three wave mixing process in a nonlinear system, where one of the waves is an electric signal with frequency $\Omega$ and spatial frequency q that modulates the refractive index [15]. However, while the modulation can be designed to match the mode separation $(\Omega, q)$ for a particular optical frequency $\omega_{0}$ in principle, the matching no longer exists for neighbouring frequencies $\omega_{0}+\Delta \omega$ if the bands involved in the transition are not completely parallel throughout the desired frequency bandwidth [2-4]. There exists, thus, an intrinsic limitation on the bandwidth over which a transition is possible, imposed by the difference in the slopes of the bands, thus their group velocity mismatch. There are other practical limits: the frequency shift $\Omega$ is limited by the achievable modulation frequencies to a maximum of tens of $\mathrm{GHz}$, and the typical wave vector difference q requires a spatial periodicity of the modulation that must be encoded in the geometry of the device, since it cannot be matched by the spatial periodicity of a travelling GHz electric signal [4]. Thus, tuning of the indirect photonic transition is only possible by varying the geometry of the device.

Quasi-indirect photonic transitions in a photonic crystal waveguide have now also been demonstrated in a two-step process, where the frequency shift is realized first, followed by a shift of the wave vector [5]. This approach is still applicable for tunable delay but does not have all the properties of the indirect transition. It cannot, for example, transfer light to a state of zero group velocity or produce large frequency shifts.

From microwave engineering, it is known that an indirect photonic transitions can be induced in a single step by the interaction of electromagnetic radiation with a moving perturbation [16]. This perturbation can be any kind of travelling discontinuity of the electromagnetic properties of the medium where the radiation propagates. Such a perturbation can be, for instance, a moving free-carrier plasma front in a semiconductor [17, 18], which leads to a moving refractive index front via the carrier-plasma dispersion effect [12]. Indirect photonic transitions induced upon interaction of an optical signal with a refractive index front moving along a photonic crystal waveguide have been employed to realize a tunable optical delay [14]. Furthermore, it was theoretically shown that indirect photonic transitions induced upon interaction with a mechanical shock front moving along a Bragg stack can lead to the generation of inverse Doppler shifted frequencies [19].

Here we present the theory of the indirect transition through the pulse interaction with a moving refractive index front. This transition can lead to a palette of signal pulse modifications which will be discussed in this paper. Finally we present the experimental results of our recent work on indirect transition with moving front in slow light waveguides [20, 21].

\section{THEORY OF INDIRECT TRANSITION WITH MOVING FRONT}

We consider the signal wave before and after its interaction with the front. The signal after the interaction is assumed to have a new frequency and wavevector. The relationship between the change in frequency and wave vector of the signal with respect to the moving front can be understood by first studying the process in the rest frame of the front. In this frame, the front can be chosen to lie at the position $\mathrm{z}_{\mathrm{f}}{ }^{\prime}=0$ and the frequency is conserved. If $\omega_{1}{ }^{\prime}, \mathrm{k}_{1}{ }^{\prime}, \omega_{2}{ }^{\prime}, \mathrm{k}_{2}{ }^{\prime}$ denote the frequencies and wave vectors of the signal on each side of the front, the phase difference on each side is:

$$
\left(\frac{\omega_{2}^{\prime}-\omega_{1}^{\prime}}{c}, k_{2}^{\prime}-k_{1}^{\prime}\right) \cdot\left(c t^{\prime}, z_{f}^{\prime}\right)=\left(\omega_{2}^{\prime}-\omega_{1}^{\prime}\right) t^{\prime}-\left(k_{2}^{\prime}-k_{1}^{\prime}\right) z_{f}^{\prime}=0,
$$

where $\mathrm{c}$ is the light velocity in vacuum and t' is the time coordinate in the rest frame of the front. This is a four vector product which is invariant under Lorentz transformation. Thus, the time and space dependent phase of the signal is continuous at the position of the moving front, independently of the reference frame of the observer. If we now denote 
the frequencies and wave vectors in the laboratory frame by $\omega_{1}, \mathrm{k}_{1}, \omega_{2}, \mathrm{k}_{2}$, the velocity of the refractive index front in the laboratory frame by $\mathrm{v}_{\mathrm{f}}$ and its position by $\mathrm{z}_{\mathrm{f}}$, we find:

$$
\begin{aligned}
& \left(\omega_{2}-\omega_{1}\right) t-\left(k_{2}-k_{1}\right) z_{f}=0 \\
& \frac{\omega_{2}-\omega_{1}}{k_{2}-k_{1}}=\frac{z_{f}}{t}=\frac{t \mathrm{v}_{f}}{t}=\mathrm{v}_{f}
\end{aligned}
$$

Eq. 2 indicates that, as observed in the laboratory frame, the ratio of the changes of frequency and wave vector induced by the interaction with the moving front is identical to the velocity at which the front propagates.

In order to determine the magnitude of the indirect photonic transition induced by the front we use a graphical representation known from microwave research $[22,23]$ and shown in Fig. 1b. The lower solid and the upper dashed curves in Fig. 1b schematically show the dispersion bands of a silicon photonic crystal waveguide for silicon refractive indices $\mathrm{n}_{\mathrm{si}}$ and $\mathrm{n}_{\mathrm{si}}+\Delta \mathrm{n}_{\mathrm{FC}}$, respectively. At the input of the structure, the signal pulse travels in a waveguide with silicon refractive index of $n_{s i}$, and is represented by a point $\left(\omega_{1}, \mathrm{k}_{1}\right)$ lying on the corresponding dispersion curve. Next, we draw all points $(\omega, \mathrm{k})$ which fulfill the phase continuity condition from Eq. 2 by plotting a straight phase continuity line with a slope equal to the travelling velocity of the front $\mathrm{v}_{\mathrm{f}}$. After being overtaken by the refractive index front, the signal pulse travels in a waveguide with silicon refractive index of $\mathrm{n}_{\mathrm{si}}+\Delta \mathrm{n}_{\mathrm{FC}}$. Its final frequency and wave vector $\left(\omega_{2}, \mathrm{k}_{2}\right)$ are determined graphically from the crossing point of the grey phase continuity line and the upper dashed dispersion curve.
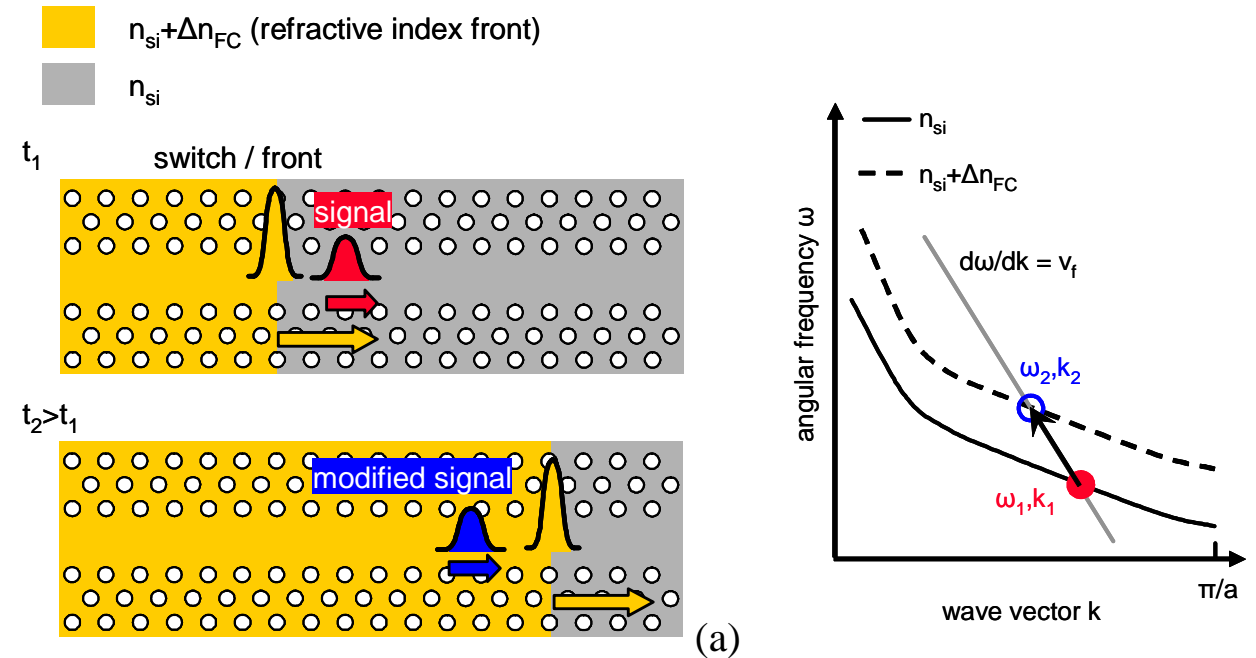

(a)

Figure 1. (a) Schematic representation of the experiment performed in [20]. A switching pulse with high peak power generates free carriers in the silicon by two-photon absorption and consequently induces a change of refractive index whose front propagates with the velocity of the switching pulse. Here, the switching pulse is faster than the signal. (b) Schematic representation of the indirect photonic transition induced.

Using this graphical representation, one easily recognizes fundamental differences from the concepts discussed before, where the refractive index is modulated with fixed temporal and spatial modulation frequencies [1-4]. In our case, the velocity of the refractive index front does not unambiguously determine one, but rather a whole set of possible final states $(\omega, \mathrm{k})$. Out of this set, such transitions will take place for which the final state corresponds to a photonic mode of the system. As long as this second condition is satisfied, there are no intrinsic limitations on the bandwidth over which a perfect mode frequency and wave vector matching can be achieved. Furthermore, the velocity of the refractive index front, which is identical to the velocity of the switching pulse, can be tuned via the operating point of the slow-light waveguide. Thus, the indirect photonic transitions are tunable without the need for any geometrical modifications. 


\section{POSSIBLE TRANSITIONS}

As will be show later, our experimental results confirm the theoretical prediction that the magnitude of the induced transitions depends on the propagation velocity of the front and on the photonic bands of the structure, as represented in Fig. 1b. Therefore, by exploiting the flexibility in dispersion design provided by slow-light waveguides [24, 25], our results open up new versatile possibilities for light control, including non-reciprocal optical isolation, tunable optical frequency shifts, optical bandwidth compression and broadening, and tunable optical delays. For instance, the authors have proposed a theoretical concept for broadening or compressing the bandwidth of an optical signal upon reflection from a moving front of a photonic crystal [26].

Some of the other possible effects are presented in Fig. 2. Effects such as light stopping, signal reversal, large frequency shifts can all be predicted. The realisation of these effects depends on the velocity of the front and the dispersion relation of the medium or waveguide where the front and signal are propagating.

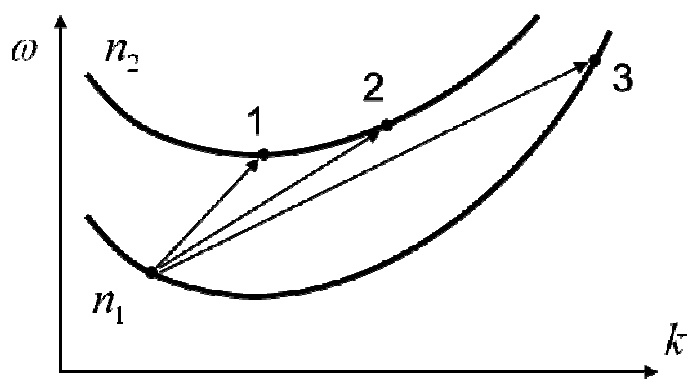

Figure 2. Schematic presentation of different effects achieved with indirect transition close to the band edge. Initial group velocities of the signal and of the front are counter directed. The velocity of the front defines the final frequency and the direction of propagation of the modified signal. The dispersion of the initial $\mathrm{n}_{1}$ medium and final $\mathrm{n}_{2}$ medium is shown. Depending on the group velocity, the point of zero group velocity (1) can be reached, thereby effectively stopping the light. Point (2) corresponds to a reversed signal propagating behind the front in the final medium. Transition (3) describes the reflection of the front with a large frequency shift.

\section{FREQUENCY SHIFT IN SLOW LIGHT WAVEGUIDES}

We have, for the first time, experimentally demonstrated indirect photonic transitions that are driven by a refractive index front travelling along a slow-light photonic crystal waveguide [20]. Fig. 3a shows a schematic representation of the experiment we performed. A high power switching pulse propagating through a silicon photonic crystal waveguide generates free carriers by two-photon absorption, and induces a corresponding change of the refractive index $\Delta \mathrm{n}_{\mathrm{FC}}$ due to the free-carrier plasma dispersion effect [12]. Accordingly, a refractive index front moves with the group velocity of the switching pulse and interacts with a slower signal co-propagating in the waveguide. Here, the difference between the group velocities of the front and the signal is chosen to be large compared to the pulse duration and the propagation length, such that the refractive index front completely overtakes the signal. The group velocity of both pulses is chosen by the frequency of light. Due to dispersion of the photonic crystal waveguide group indices from 10 to 30 are accessible.

In Fig. 3, the schematic transition and the experimental results are presented for the case when the signal is faster than the switching pulse. The signal overtakes the front and comes from the area with the refractive index shifted by the free carriers into the area without refractive index change. The schematic presentation of the indirect transition in Fig. 3(a) predicts a blue shift of the signal frequency, which cannot be obtained by the direct transition. It should be mentioned that we transfer signal from the medium with free carriers to a medium without free carriers. Thus, the direct transition in this case would produce a strong red shift, according to the relation $\Delta \omega \sim-\Delta \mathrm{n}$. Fig. 3(b) presents the wavelength shift as the function of the initial delay between the signal and the switching pulse. The dynamic transition takes place only at the delays when the signal has time to overtake the switching pulse within the dimensions of the slow light waveguide. A clear blue shift is observed. 


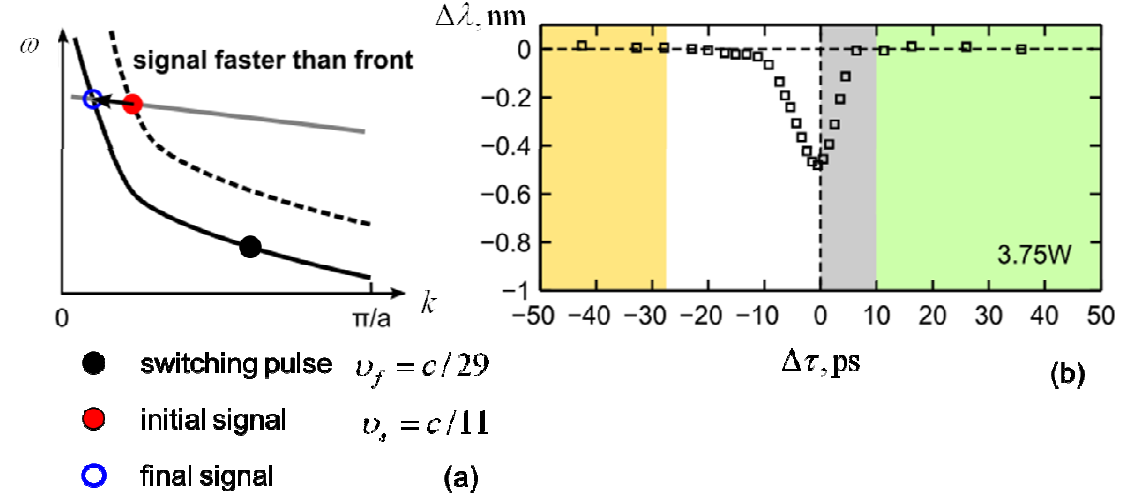

Figure 3. (a) Schematic presentation of the indirect transition when signal is faster than front. A blue shift is expected from the band diagram. (b) Blue shift measured as the delay variation between signal and switching pulse. This measurement supports the indirect transition prediction.

\section{CONCLUSION}

We have presented the theory of indirect photonic transitions obtained by the pulse interaction with the refractive index front. Different effects such as frequency shift, light stopping and time reversals are predicted. Experimental demonstration of the frequency shift in the slow light waveguide is presented, where the front is realized as a free carrier injection caused by a high power switching pulse. The effect of indirect transition is confirmed by the measurement.

\section{ACKNOWLEDGEMENTS}

This research was supported by the German Research Foundation DFG (EI 391/12-2). The authors would like acknowledge the support from CST, Darmstadt, Germany with their Microwave Studio software.

\section{REFERENCES}

[1] J. N. Winn, S. Fan, J. D. Joannopoulos, and E. P. Ippen, "Interband transitions in photonic crystals," Phys. Rev. B 59, 1551-1554 (1999).

[2] Z. Yu and S. Fan, "Complete optical isolation created by indirect interband photonic transitions," Nature Photon. 3, 91-94 (2009).

[3] Z. Yu and S. Fan, "Integrated Nonmagnetic Optical Isolators Based on Photonic Transitions," IEEE J. Sel. Top. Quantum Electron. 16, 459-466 (2010).

[4] H. Lira, Z. Yu, S. Fan, and M. Lipson, "Electrically Driven Nonreciprocity Induced by Interband Photonic Transition on a Silicon Chip," Phys. Rev. Lett. 109, 33901 (2012).

[5] D. M. Beggs, I. H. Rey, T. Kampfrath, N. Rotenberg, and L. Kuipers, et al., "Ultrafast Tunable Optical Delay Line Based on Indirect Photonic Transitions," Phys. Rev. Lett 108, 213901 (2012).

[6] M. Notomi and S. Mitsugi, "Wavelength conversion via dynamic refractive index tuning of a cavity," Phys. Rev. A 73, 51803 (May, 2006).

[7] S. F. Preble, Q. Xu, and M. Lipson, "Changing the colour of light in a silicon resonator," Nature Photon. 1, 293-296 (May, 2007).

[8] M. Castellanos Muñoz, A. Y. Petrov, and M. Eich, "All-optical on-chip dynamic frequency conversion," Appl. Phys. Lett. 101, 141119-4 (2012).

[9] J. Upham, Y. Tanaka, T. Asano, and S. Noda, "On-the-Fly Wavelength Conversion of Photons by Dynamic Control of Photonic Waveguides," Appl. Phys. Express 3, 62001 (2010).

[10] T. Kampfrath, D. M. Beggs, T. P. White, A. Melloni, and T. F. Krauss, et al., "Ultrafast adiabatic manipulation of slow light in a photonic crystal," Phys. Rev. A 81, 43837 (April, 2010).

[11]I. Geltner, Y. Avitzour, and S. Suckewer, "Picosecond pulse frequency upshifting by rapid free-carrier creation in ZnSe," Appl. Phys. Lett 81, 226-228 (2002). 
[12] R. Soref and B. Bennett, "Electrooptical effects in silicon," IEEE J. Quantum Electron. 23, 123-129 (January, 1987).

[13] T. Tanabe, E. Kuramochi, H. Taniyama, and M. Notomi, "Electro-optic adiabatic wavelength shifting and Q switching demonstrated using a p-i-n integrated photonic crystal nanocavity," Opt. Lett. 35, 3895-3897 (December, 2010).

[14] K. Kondo, M. Shinkawa, Y. Hamachi, Y. Saito, and Y. Arita, et al., "Ultrafast Slow-Light Tuning Beyond the Carrier Lifetime Using Photonic Crystal Waveguides," Phys. Rev. Lett 110, 53902 (2013).

[15]R. Stolte and R. Ulrich, "Integrated-optical gigahertz frequency shifter for 1.5 um signals," Electron. Lett. 33, 1217-1219 (1997).

[16] M. Lampe, E. Ott, and J. H. Walker, "Interaction of electromagnetic waves with a moving ionization front," Phys. Fluids 21, 42-54 (1978).

[17]D. Hashimshony, A. Zigler, and K. Papadopoulos, "Conversion of Electrostatic to Electromagnetic Waves by Superluminous Ionization Fronts," Phys. Rev. Lett 86, 2806-2809 (2001).

[18] J. Bae, Y. J. Xian, S. Yamada, and R. Ishikawa, "Doppler frequency up conversion of electromagnetic waves in a slotline on an optically excited silicon substrate," Appl. Phys. Lett 94, 91120-3 (2009).

[19]E. J. Reed, M. Soljacic, and J. D. Joannopoulos, "Reversed Doppler effect in photonic crystals," Phys. Rev. Lett. 91, 133901 (2003).

[20] M. Castellanos Muñoz, A. Y. Petrov, L. O'Faolain, J. Li, T. F. Krauss, and M. Eich, "Optically Induced Indirect Photonic Transitions in a Slow Light Photonic Crystal Waveguide", Phys. Rev. Lett., 112, 053904 (2014).

[21] M. Castellanos Muñoz, A. Y. Petrov, L. O'Faolain, J. Li, T. F. Krauss, and M. Eich, "All-optical dynamic frequency conversion in silicon photonic crystals," IEEE 10th International Conference on Group IV Photonics (GFP), 162 (2013).

[22] W. B. Mori, T. Katsouleas, J. M. Dawson, and C. H. Lai, "Conversion of dc Fields in a Capacitor Array to Radiation by a Relativistic Ionization Front," Phys. Rev. Lett 74, 542-545 (1995).

[23] N. Seddon and T. Bearpark, "Observation of the Inverse Doppler Effect," Science 302, 1537-1540 (2003).

[24] A. Y. Petrov and M. Eich, "Zero dispersion at small group velocities in photonic crystal waveguides," Appl. Phys. Lett. 85, 4866-4868 (November, 2004).

[25] J. Li, T. P. White, L. O'Faolain, A. Gomez-Iglesias, and T. F. Krauss, "Systematic design of flat band slow light in photonic crystal waveguides," Opt. Express 16, 6227-6232 (April, 2008).

[26]E. Ulchenko, D. Jalas, A. Y. Petrov, M. Castellanos Muñoz, S. Lang, and M. Eich, "Pulse compression and broadening by reflection from a moving front of a photonic crystal," submitted to Opt. Express (2014). 